\title{
Osteoprogenitor differentiation is not affected by immunomodulatory thalidomide analogs but is promoted by low bortezomib concentration, while both agents suppress osteoclast differentiation
}

\author{
SHOSO MUNEMASA ${ }^{1}$, AKIRA SAKAI ${ }^{1}$, YOSHIAKI KURODA ${ }^{1}$, YOSHIKO OKIKAWA ${ }^{1}$, YUTA KATAYAMA ${ }^{3}$, \\ HIDEKI ASAOKU $^{3}$, TADAHIKO KUBO ${ }^{2}$, SHOJI SHIMOSE $^{2}$ and AKIRO KIMURA ${ }^{1}$
}

Departments of ${ }^{1}$ Hematology and Oncology, RIRBM, ${ }^{2}$ Orthopedic Surgery, Hiroshima University;

${ }^{3}$ Internal Medicine, Hiroshima Red-Cross Hospital, Hiroshima, Japan

Received February 19, 2008; Accepted April 4, 2008

\begin{abstract}
We investigated the effects of bortezomib (PS$341)$ and immunomodulatory thalidomide analogs (immunomodulatory compounds; CC-4047, CC-6032, and CC-5013, or lenalidomide) on osteoblast and osteoclast differentiation in vitro using human mesenchymal stem cells (hMSC) to commit to osteoprogenitor cells and peripheral blood mononuclear cells (PBMCs) isolated from healthy donors, respectively. First, the concentration of bortezomib for an anti-myeloma effect was more than $1.0 \mathrm{nM}$ in myeloma cells of multiple myeloma (MM) patients and more than $2.5 \mathrm{nM}$ in myeloma cell lines. In contrast, anti-myeloma effects of immunomodulatory compounds on myeloma cells differed among myeloma cells and these compounds themselves. Subsequently, these agents (bortezomib; 0.5-5.0 nM, immunomodulatory compounds; $10 \mu \mathrm{M}$ ) were added to the osteoprogenitor cell culture media or the media for osteoclast differentiation. Low bortezomib concentrations (0.5 and 1.0 $\mathrm{nM}$ ) increased ALP activity, and the delayed addition of bortezomib further increased ALP activity. Mineralized nodular formation with $<2.5 \mathrm{nM}$ bortezomib was not impaired. BMP2 expression on osteoprogenitor cells was found to increase in a time-dependent manner irrespective of treatment with bortezomib. On the other hand, the antiosteoclast effect with low bortezomib concentration $(\leq 2.5 \mathrm{nM})$ depended on MM patients. In contrast, immunomodulatory compounds at $10 \mu \mathrm{M}$ showed an anti-osteoclast effect without cytotoxicity to osteoblast differentiation, at which
\end{abstract}

Correspondence to: Dr Akira Sakai, Department of Hematology and Oncology, RIRBM, Hiroshima University, 1-2-3 Kasumi, Minami-ku, Hiroshima 734-8553, Japan

E-mail: sakira@hiroshima-u.ac.jp

Key words: thalidomide, bortezomib, osteoclast differentiation dose myeloma cells underwent apoptosis. These findings might improve the treatment strategy for MM patients without damaging BM stromal cells by combining bortezomib with immunomodulatory compounds.

\section{Introduction}

Bone disease (BD) is a frequent characteristic of multiple myeloma (MM) and leads to severe clinical complications. The severity of BD correlates with the tumor burden and is one of the major parameters in the Durie and Salomon clinical staging system (1). BD in MM is due to not only the activation of osteoclasts but also the impairment of osteoblast differentiation. Therefore, osteoclast-activating factors (OAF), such as interleukin-1ß (IL-1ß) (2), macrophage inflammatory protein-1 $\alpha$ (MIP-1 $\alpha$ ) and MIP-1 $1 \beta$ produced by myeloma cells (3), or receptor activator of NF-кB ligand (RANKL) expressed on stromal cells contacted with myeloma cells $(4,5)$, play key roles in the development activation of osteoclasts in MM. On the other hand, overexpression of the Wnt signaling antagonists dickkopf1 (DKK1) and FRZB (secreted Frizzledrelated protein 2, sFRP-2) correlates with MM-related bone disease $(6,7)$. This combination of bone destruction and impairment of bone restoration is thought to be the cause of $\mathrm{BD}$ in MM.

Treatment for MM using new drugs, such as bortezomib (PS-341), thalidomide, and lenalidomide, has improved overall survival (OS). Interestingly, some reports have shown high serum bone-specific alkaline phosphatase levels after bortezomib-combined therapy in MM patients $(8,9)$, and administration of proteasome inhibitor induced bone formation accompanied with the expression of bone morphogenetic protein-2 (BMP-2) in mice (10). However, the effect of bortezomib on osteoclast differentiation and the effects of immunomodulatory compounds on osteoclast or osteoblast differentiation remain unknown.

Here we analyzed the effects of these new drugs, bortezomib and immunomodulatory compounds, which have attracted a great deal of attention in the treatment of MM, on osteoclast and osteoblast differentiation in vitro. 


\section{Materials and methods}

Reagents. Bortezomib (PS-341) and immunomodulatory thalidomide analogs (immunomodulatory compounds; CC4047, CC-6032 and CC-5013, or lenalidomide) were kindly donated by Millennium Pharmaceuticals Inc. (Cambridge, MA, USA) and Celgene Corporation (Warren, NJ, USA), respectively. They were dissolved in dimethylsulfoxide (DMSO) and stored at $-20^{\circ} \mathrm{C}$. The final concentration of DMSO in all experiments was $<0.1 \%$.

Myeloma cell purification. Bone marrow (BM) mononuclear cells (MNCs) were isolated from fresh BM aspirates as described previously (11). Plasma (myeloma) cells were purified using MACS CD138 Micro Beads (Miltenyi Biotec, Auburn, CA, USA) according to the manufacturer's instructions. Written informed consent was obtained from all patients.

Cell proliferation assay. To evaluate the cytotoxic effects of bortezomib and immunomodulatory compounds on myeloma cell lines (U266, KMS21BM, and RPMI8226) and purified myeloma cells of MM patients, a Cell Counting Kit-8 (Dojindo Laboratories, Kumamoto, Japan) was used according to the manufacturer's instructions. This assay is based on colorimetric quantification of NADH (12). Cells were suspended in Roswell Park Memorial Institute (RPMI)-1640 medium (Nissui, Tokyo, Japan) supplemented with $10 \%$ fetal calf serum (FCS; M.A. Bioproducts, Walkersville, MD, USA), and seeded at $5 \times 10^{4}$, unless otherwise indicated, in a final volume of $100 \mu 1$ (10\% FCS) in 96-well flat-bottomed plates (Corning, NY, USA). Plates were incubated at $37^{\circ} \mathrm{C}$ in a $5 \%$ $\mathrm{CO}_{2}$ incubator for 1-3 days. At the end of incubation, $10 \mu \mathrm{l}$ of WST-8 reagent was added to each well and the plates were incubated for $4 \mathrm{~h}$. Absorbance was then measured using an ImmunoMini NJ-2300 plate reader (Nalge Nunc International, Rochester, NY, USA).

Human mesenchymal stem cell (hMSC) culture to commit to osteoprogenitor cells. The hMSC were obtained from SankoJunyaku Co. (Tokyo, Japan), and cultured in Mesenchymal Stem Cell Basal Media (MSCBM) containing mesenchymal cell growth supplement (MCGS), penicillin-streptomycin, and L-glutamine (SankoJunyaku Co.). After reaching 90\% confluence, the medium was changed from MSCBM to hMSC Differentiation Basal Medium-Osteogenic containing MCGS, dexamethasone, L-glutamine, ascorbate, penicillinstreptomycin, and B-glycerophosphate (SankoJunyaku Co.). To evaluate the effects of bortezomib and immunomodulatory compounds (CC-4047, CC-6032, CC-5013) on osteoblast differentiation, these components were added to the culture media according to their purpose. Media containing bortezomib (10-0.5 nM) were replenished twice a week, and immunomodulatory compounds $(10 \mu \mathrm{M})$ were added every day. As controls for bortezomib and immunomodulatory compounds, media were supplemented with $<0.1 \%$ DMSO.

Alkaline phosphatase activity assay. At 7 and 16 days after the initiation of osteoprogenitor cell culture, the cells were washed twice with ice-cold phosphate-buffered saline (PBS), and incubated with $200 \mu 11 \mathrm{X}$ Passive Lysis Buffer (Promega). After gentle shaking for $15 \mathrm{~min}$ at room temperature, the cells were scrapped off and centrifuged at $1000 \mathrm{~g}$ (12000 rpm) for $5 \mathrm{~min}$. Protein concentration of the supernatants was assayed using a Micro $\mathrm{BCA}^{\mathrm{TM}}$ Protein assay kit (Pierce Chemical Co., Rockford, USA). Alkaline phosphatase activity was analyzed using an Alkaline Phosphatase Opt Kit (Roche Molecular Biochemicals Inc., Tokyo, Japan). ALP activity was expressed per $1 \mu \mathrm{g}$ of protein.

von Kossa staining. For mineralized nodule formation, osteoblastic cells were cultured for up to 42 days after the initiation of osteoprogenitor cell culture using hMSC Differentiation Basal Medium-Osteogenic. The cells were fixed with $20 \%$ formaldehyde for $1 \mathrm{~h}$ and washed three times with PBS. Fixed cells were incubated with $5 \%$ silver nitrate solution and exposed to sunlight for $45 \mathrm{~min}$, washed twice with deionized water, and then treated with $5 \%$ sodium thiosulfate followed by washing in flowing water. Examinations were performed in duplicate.

Osteoclast differentiation from osteoclast precursors or human bone marrow cells. Peripheral blood mononuclear cells (PBMCs) were isolated from 2 healthy donors as described previously (11). Subsequently, we deleted adherent cells by incubation in flasks for $4 \mathrm{~h}$. Non-adherent PBMCs were seeded on Lab Tec-Chamber slides (Nalge Nunc International, Rochester, NY, USA) and incubated in osteoclast precursor growth medium containing fetal bovine serum (FBS), L-glutamine, penicillin-streptomycin (SankoJunyaku Co.), and recombinant human M-CSF (rhM-CSF) $(10 \mathrm{ng} / \mathrm{ml})$ (R\&D, Minneapolis, MN, USA) for 2 days. Subsequently, we cultured the cells according to the method of Takayanagi et al (13). Briefly, cells were cultured in $\alpha$-MEM (Gibco, Invitrogen, Tokyo, Japan) containing $10 \%$ FCS, penicillinstreptomycin, rhM-CSF (10 ng/ml), and recombinant human RANKL (rhRANKL) $(50 \mathrm{ng} / \mathrm{ml})(\mathrm{R} \& \mathrm{D})$. To evaluate the effect of bortezomib (PS-341) and immunomodulatory compounds (CC-4047, CC-6032, CC-5013, or lenalidomide) on osteoclast differentiation, these components were added to the culture media according to purpose. Media containing bortezomib (PS-341) (10-1.0 nM) were replenished twice a week, and immunomodulatory compounds $(10 \mu \mathrm{M})$ were added every day. As a control for PS-341 and immunomodulatory compounds, the media were supplemented with $<0.1 \%$ DMSO.

TRAP staining. TRAP staining was performed at day 14 using a TRAP staining kit (Sigma-Aldrich, Saint Louis, MO, USA) according to the manufacturer's instructions. We counted TRAP $^{+}$multinucleated cells $(>2)$ as mature osteoclasts. Examinations were performed in duplicate.

Bone morphogenetic protein 2 (BMP2) expression by realtime quantitative reverse-transcriptase-polymerase chain reaction $(R Q-P C R)$. To evaluate the induction of $\mathrm{BMP} 2$ by bortezomib (PS-341), hMSCs were obtained from Sanko Junyaku Co. and cultured as described above. After reaching $90 \%$ confluence, the medium was changed as described above. To evaluate whether bortezomib (PS-341) induces 
(A)

(B)

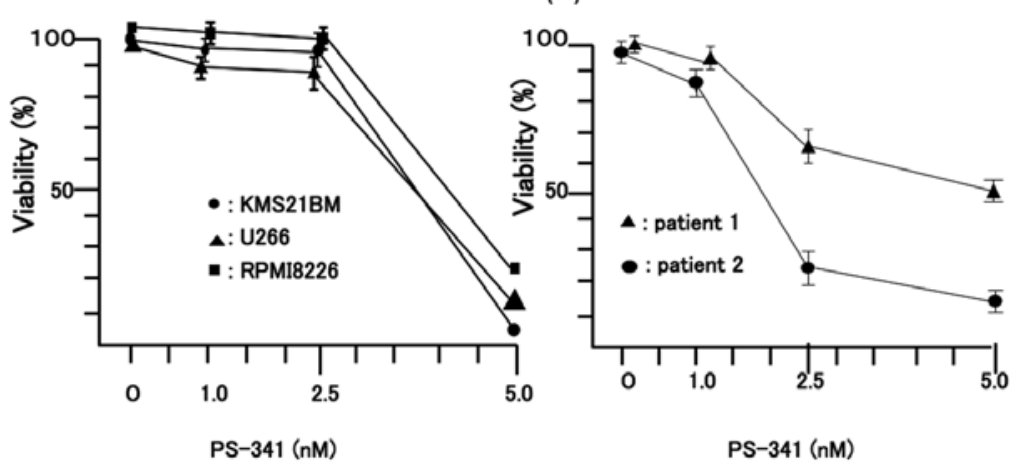

Figure 1. Effects of bortezomib (PS-341) on apoptosis in MM cells. Dose-dependent survival curves based on cell proliferation assay were generated for MM cells cultured for $24 \mathrm{~h}$ with bortezomib (0-5.0 $\mathrm{nM}$ ). (A) MM cell lines; (B) patients myeloma cells. Results are expressed as the means \pm SEM of triplicate experiments.

BMP2 and whether this effect depends on the time of addition of bortezomib to the culture media or the duration of culture, we added $1.0 \mathrm{nM}$ bortezomib (PS-341) to media at the same time as the initiation of osteoprogenitor cell culture or at 5 days after culture in this medium. Cells were incubated for 12 or $60 \mathrm{~h}$, then detached by $0.5 \%$ Trypsin-EDTA treatment and washed twice with ice-cold phosphate-buffered saline (PBS). Subsequently, total RNA was extracted using an RNeasy Mini kit (Qiagen, Tokyo, Japan) according to the manufacturer's instructions. As a control, total RNA was extracted from mesenchymal stem cells cultured in MSCBM. cDNAs were made using a first-strand cDNA synthesis kit (Amersham Pharmacia Biotech, Piscataway, NJ, USA) according to the manufacturer's instructions. RQ-PCR reactions were performed using the ABI PRISM 7700 Sequence Detection System (Applied Biosystems, Foster City, CA, USA). Primers and probes were as follows: BMP2 probe, 5'-FAM-ATCTGAACTCCACTAATC-MGB-3', BMP2 forward, 5'-GCCCTTTTCCTCTGGCTGAT-3', and BMP2 reverse, 5'-TGACCAACGTCTGAACAATGG-3'. PCRs for GAPDH were performed with a ready-made mix of reagents from Applied Biosystems (item no. 4326317E).

Statistical analysis. The significance of the association of ALP activities or the number of TRAP-positive cells under different culture conditions was assessed with the unpaired t-test. The level of significant difference by the unpaired t-test was set at $\mathrm{p}<0.05$.

\section{Results}

Effects of bortezomib (PS-341) on apoptosis in MM cells. We first tried to determine the working concentration of bortezomib in our culture system of osteoblast and osteoclast differentiation by treatment of MM cell lines (KMS21BM, U266, and RPMI8226) with bortezomib (0-5.0 nM). As seen in Fig. 1, treatment of MM cells with $5 \mathrm{nM}$ bortezomib for $24 \mathrm{~h}$ induced a significant $(\mathrm{p}<0.05)$ decrease of viability in these cell lines. Low bortezomib concentrations of not more than $2.5 \mathrm{nM}$ did not show any apoptotic effect on these MM cell lines. On the other hand, treatment of patient myeloma cells with $2.5 \mathrm{nM}$ bortezomib for $24 \mathrm{~h}$ induced a significant $(\mathrm{p}<0.05)$ decrease of viability. This result led us to use a concentration of bortezomib of $\leq 5.0 \mathrm{nM}$ for analysis of the effects on osteoblast and osteoclast differentiation.

Effects of immunomodulatory thalidomide analogs (immunomodulatory compounds: CC-4047, CC-6032, CC-5013 or lenalidomide) on apoptosis in MM cells. Next we tried to determine the working concentrations of immunomodulatory compounds as mentioned in Fig. 1. As shown in Fig. 2, MM cells were treated with the immunomodulatory compounds $(0-10 \mu \mathrm{M})$ for $72 \mathrm{~h}$. Treatment of $\mathrm{U} 266$ with $1 \mu \mathrm{M}$ immunomodulatory compounds (all compounds) induced a significant $(\mathrm{p}<0.05)$ decrease of viability; however, treatment with 5.0 and $10 \mu \mathrm{M}$ did not induce any further decrease (Fig. 2A). Treatment of KMS21BM with CC-6032 and CC-5013 induced a significant $(\mathrm{p}<0.05)$ decrease of viability in a dose-dependent manner, and treatment with $1 \mu \mathrm{M}$ CC-4047 induced a significant $(\mathrm{p}<0.05)$ decrease of viability. However, treatment with 5.0 and $10 \mu \mathrm{M} \mathrm{CC}-4047$ did not induce a further decrease (Fig. 2B). Furthermore, treatment of RPMI8226 with CC-6032 showed a dose-dependent anti-myeloma cell effect; however, treatment with CC-5013 did not show an antimyeloma cell effect at any concentration against RPMI8226, and treatment with $\mathrm{CC}-4047$ induced a significant $(\mathrm{p}<0.05)$ decrease of viability only at $10 \mu \mathrm{M}$ (Fig. 2C). Treatment of patient myeloma cells with 1 M CC-6032 and CC-5013 induced a significant $(\mathrm{p}<0.05)$ decrease of viability; however, treatment with CC-4047 did not induce a significant decrease of viability at $1 \mu \mathrm{M}$, and treatment with 5.0 and $10 \mu \mathrm{M}$ of these agents did not induce a further decrease (Fig. 2D). Therefore, the apoptotic effects of immunomodulatory compounds on myeloma cells differed among myeloma cells and immunomodulatory compounds themselves.

Low bortezomib (PS-341) concentration promotes osteoblast differentiation. Recent reports showing high serum bonespecific alkaline phosphatase level after bortezomibcombined therapy in MM patients led us to expect that a low concentration of bortezomib would support osteoblast differentiation. Here we used a human mesenchymal stem cell (hMSC) culture system to analyze whether bortezomib promotes osteoprogenitor cell differentiation according to 

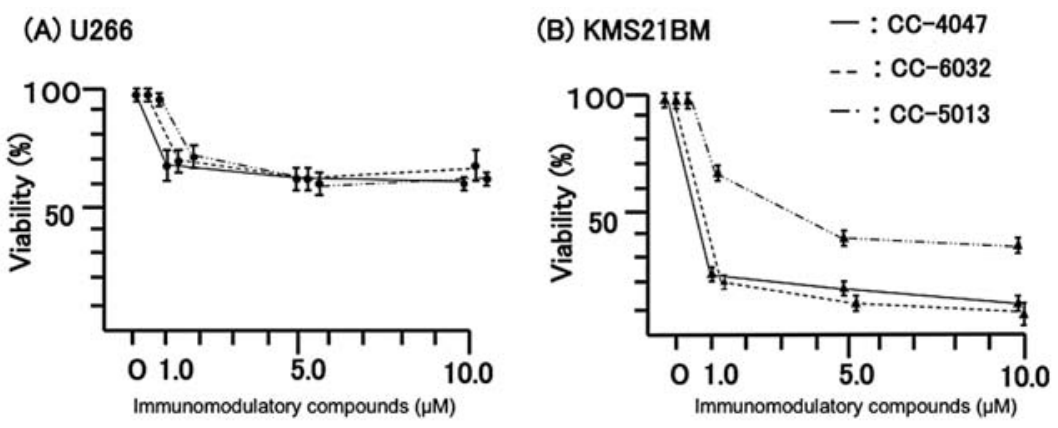

(C) RPMI8226

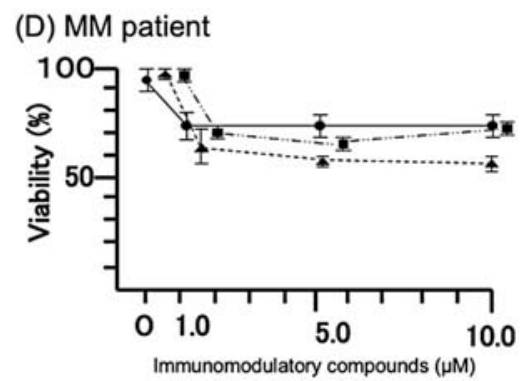

Figure 2. Effects of immunomodulatory thalidomide analogs (immunomodulatory compounds; CC-4047, CC-6032, CC-5013) on apoptosis in MM cells. Dosedependent survival curves based on cell proliferation assay were generated for MM cells cultured for $72 \mathrm{~h}$ with immunomodulatory compounds $(0-10.0 \mu \mathrm{M})$. No addition of immunomodulatory compounds for $72 \mathrm{~h}$ after the initial medium. Results are expressed as the means \pm SEM of triplicate experiments.

concentration. The fate of commitment from hMSC depends on the medium, so we used medium for osteoprogenitor cell culture after reaching $90 \%$ confluence. First, we added bortezomib $(0.5-10 \mathrm{nM})$ to the media from the initiation of osteoprogenitor cell culture. As shown in Fig. 3A, ALP activity was significantly different and dose-dependent; $5.0 \mathrm{nM}$ bortezomib showing an anti-myeloma cell effect also suppressed ALP activity in osteoprogenitor cells, $<2.5 \mathrm{nM}$ bortezomib did not suppress it compared with the control. The effective concentration of bortezomib in sera of MM patients is not well known. The $\mathrm{IC}_{50}(\mathrm{nM})$ was reported to be 2.5-30 nM in myeloma cells of MM patients (14). Of note, ALP activity in $0.5 \mathrm{nM}$ bortezomib was significantly higher $(\mathrm{p}<0.05)$ than that of the control apart from the antimyeloma cell effect; however, there was no difference in ALP activity between 1.0 and $2.5 \mathrm{nM}$ bortezomib and the control. Next, we added bortezomib $(0.5-5.0 \mathrm{nM})$ to the media 5 days after the initiation of osteoprogenitor cell culture to investigate whether the initiation of bortezomib would affect the ALP activity. As shown in Fig. 3A, ALP activity was significantly $(\mathrm{p}<0.05)$ higher in culture medium with the addition of 5 days of delayed bortezomib except for the concentration of $0.5 \mathrm{nM}$, and there were no significant differences in ALP activity of osteoprogenitor cells cultured between $1.0 \mathrm{nM}$ and $0.5 \mathrm{nM}$ bortezomib. Therefore, these results showed that a low bortezomib concentration could promote osteoprogenitor cell differentiation and might be more effective for slightly matured osteoprogenitor cells than immature cells. Next, we investigated mineralized nodule formation by von Kossa staining at 42 days after the initiation of osteoprogenitor cell culture simultaneously with bortezomib (1.0-10 nM). As shown in Fig. 3B, $10 \mathrm{nM}$ bortezomib completely suppressed mineralized nodule formation; however, we detected mineralized nodule formation in osteoblasts incubated with $5.0 \mathrm{nM}$ bortezomib, in which the anti-myeloma cell effect was confirmed in Fig. 1, and the mineralized nodule formation with 2.5 and $1.0 \mathrm{nM}$ bortezomib seemed to be similar to that of the control. These results suggested that long culture might compensate for the difference in ALP activity according to the concentration of bortezomib in the early stage of osteoblast differentiation.

Immunomodulatory thalidomide analogs (CC-4047, CC-6032, CC-5013) do not suppress osteoblast differentiation. We investigated ALP activity in osteoprogenitor cells at day 16 by adding $10 \mu \mathrm{M}$ of each immunomodulatory compound to media after the initiation of osteoprogenitor cell culture. The anti-myeloma cell effect in this concentration was confirmed in Fig. 2 and by a previous study (15). As shown in Fig. 4A, no immunomodulatory compounds suppressed ALP activity compared with the control at this concentration. Subsequently, we investigated mineralized nodule formation by von Kossa staining at day 42, and it was detected similarly in all osteoprogenitor cell cultures with immunomodulatory compounds compared with the control (Fig. 4B). These results suggested that immunomodulatory compounds would not suppress osteoblast differentiation.

BMP2 expression in osteoprogenitor cells might be timedependent in osteoprogenitor cell culture but independent of the treatment of bortezomib (PS-341). We analyzed the expression of BMP2 in osteoprogenitor cells by adding $1.0 \mathrm{nM}$ bortezomib in the early stage of osteoblast differentiation from MSCs since BMP2 is thought to have an autocrine effect on osteoblast differentiation (10) and to be an important factor in the early stage of osteogenic differentiation (16). We added $1.0 \mathrm{nM}$ bortezomib to media at the same time as the initiation of osteoprogenitor cell culture or after culture for 5 days in these media. This concentration $(1.0 \mathrm{nM})$ was 


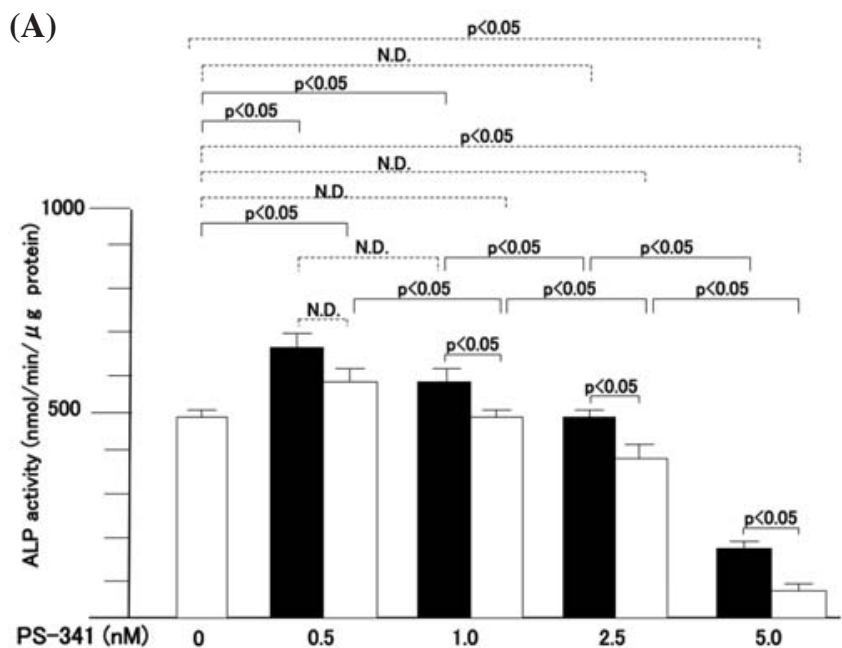

(B)

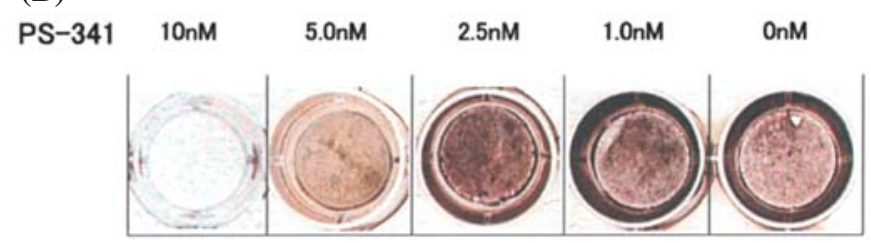

Figure 3. Low concentration of bortezomib (PS-341) promotes osteoblast differentiation. (A) ALP activity was analyzed at day 16 after the addition of different concentrations of bortezomib to the culture medium for osteoblasts from initiation of the change of medium. The highest concentration of bortezomib was $5.0 \mathrm{nM}$ since we confirmed that this concentration showed an anti-myeloma effect (Fig. 1). Then, we gradually decreased the concentration of bortezomib (5.0, 2.5, 1.0, and $0.5 \mathrm{nM}$ ) in the medium and analyzed ALP activity to compare with the control. The analysis of ALP activity between two different initiation days of bortezomib administration; white bars, bortezomib was added from initiation of the change of medium; and black bars, bortezomib was added from 5 days after the initiation of medium. Results are expressed as the means \pm SEM of triplicate experiments. (B) von Kossa staining was performed at day 42 after the initiation of culture for osteoblast differentiation. Bortezomib was added from the initiation day. Bortezomib at $10 \mathrm{nM}$ completely suppressed mineralized nodule formation and at $5.0 \mathrm{nM}$ also suppressed mineralized nodule formation. Bortezomib at $2.5 \mathrm{nM}$ showed similar mineralized nodule formation to the control.

determined based on the result showing that both 1.0 and $0.5 \mathrm{nM}$ bortezomib significantly increased ALP activity (Fig. 3B). Cells were incubated for 12 or $60 \mathrm{~h}$ after the addition of bortezomib, and total RNA was subsequently extracted. As a control, RNA was also extracted from cells incubated without bortezomib treatment. There was a significant difference $(\mathrm{p}<0.05)$ in BMP2 expression between the sample with $12 \mathrm{~h}$ and that with 5 days plus 12-h incubation in osteoprogenitor cell culture medium, and also between the sample with $60 \mathrm{~h}$ and that with 5 days plus 60-h incubation irrespective of bortezomib (Fig. 5). Although there was a significant difference $(\mathrm{p}<0.05)$ in BMP2 expression between the sample with $12 \mathrm{~h}$ and that with $60 \mathrm{~h}$ incubation with bortezomib 5 days after the initiation of osteoprogenitor cell culture, the same difference was found in control culture. There was also no difference in BMP2 expression between 12- and 60-h incubation with bortezomib from the initiation of osteoprogenitor cell culture. Furthermore, there was no difference in BMP2 expression between incubation with and without bortezomib for each duration of culture. Therefore, BMP2 mRNA content was found to increase in a time-
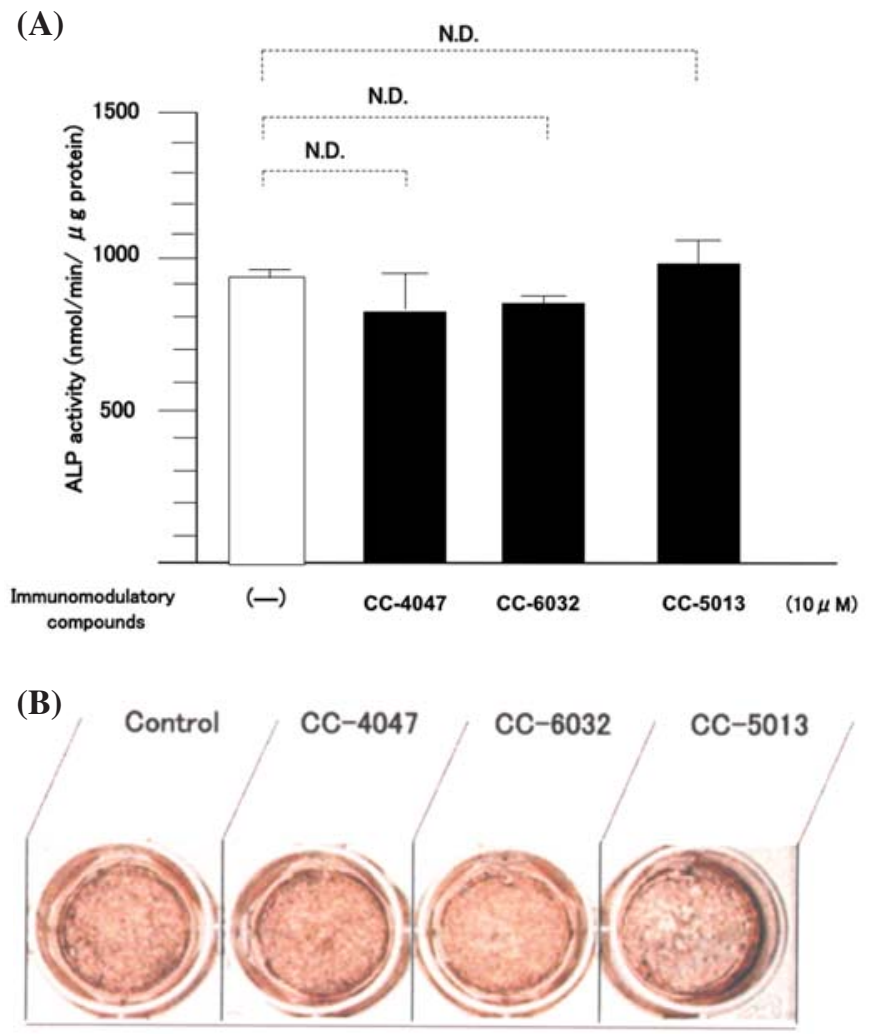

Figure 4. Immunomodulatory compounds do not suppress osteoblast differentiation. (A) ALP activity was analyzed at day 16 after the addition of $10 \mu \mathrm{M}$ of immunomodulatory compounds (CC-4047, CC-6032, and CC-5013, respectively) to the culture medium for osteoblast differentiation from initiation of the change of medium. Results are expressed as the means \pm SEM of triplicate experiments. (B) von Kossa staining was performed at day 42 after the initiation of culture for osteoblast differentiation. Immunomodulatory compounds $(10 \mu \mathrm{M})$ were added from the initiation day. All immunomodulatory compounds showed similar mineralized nodule formation to the control.

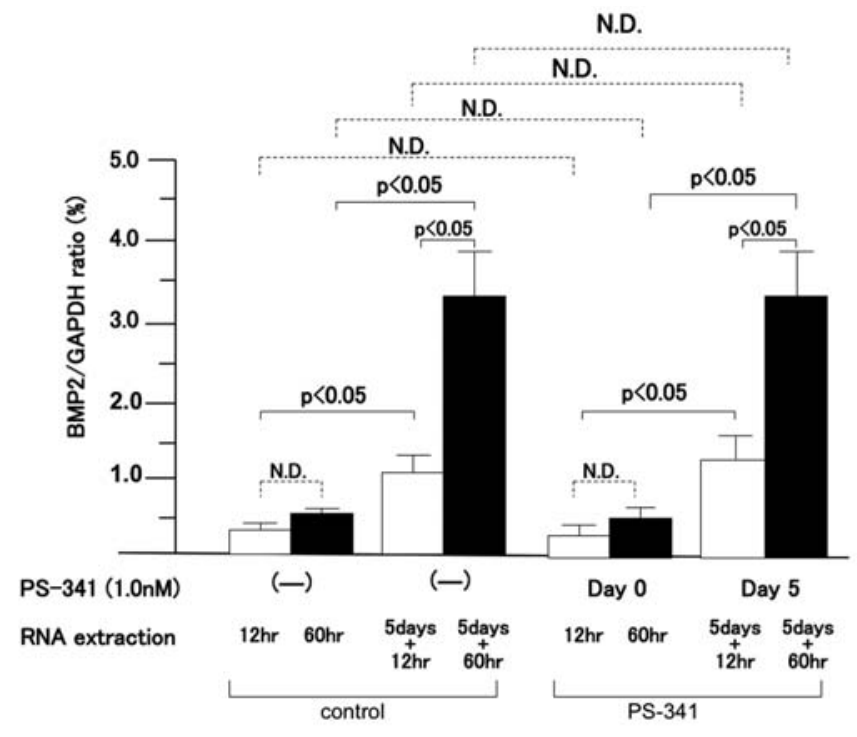

Figure 5. BMP2 expression after treatment with bortezomib (PS-341) in osteoblasts. After reaching $90 \%$ confluence of hMSC, the medium was changed for osteoblast differentiation. Bortezomib was added to medium (left) at the same time as the initiation of osteoprogenitor cell culture or (right) after culture in this medium for 5 days. Cells were incubated for 12 or $60 \mathrm{~h}$, and total RNA was extracted (white bar, $12 \mathrm{~h}$; and black bar, $60 \mathrm{~h}$ ). Results are expressed as the means \pm SEM of triplicate experiments. 
(A)
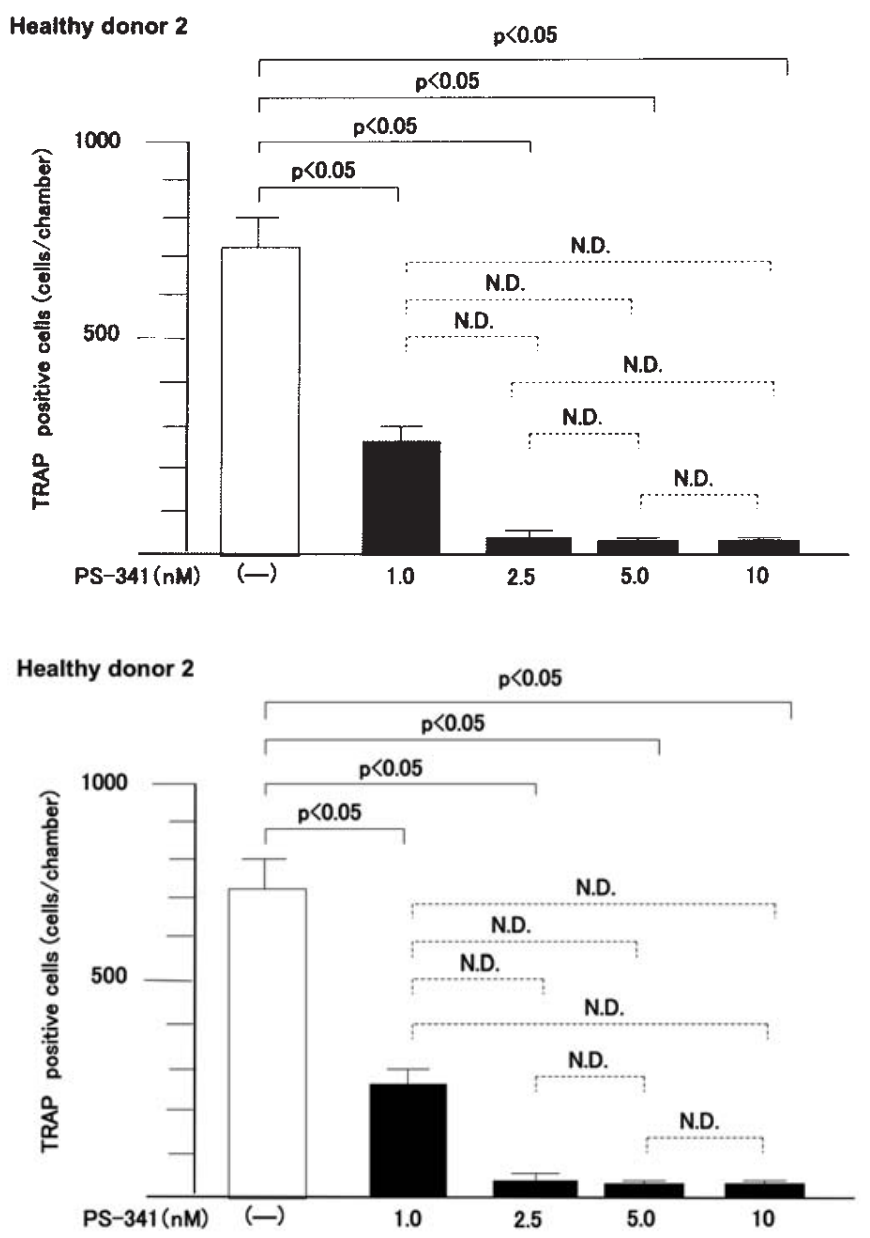

(B)

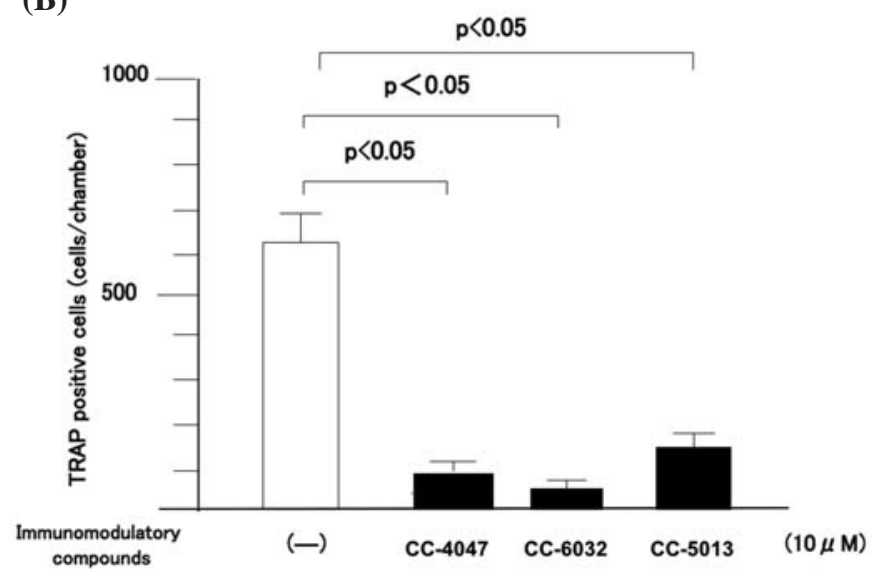

Figure 6. Both bortezomib (PS-341) and immunomodulatory thalidomide analogs suppress osteoclast differentiation. Non-adherent PBMCs from two healthy donors were seeded in Lab Tec-Chamber slides and incubated in osteoclast precursor growth medium containing recombinant rhM-CSF (10 $\mathrm{ng} / \mathrm{ml}$ ) for 2 days. Subsequently, we cultured the cells in $\alpha$-MEN containing $10 \% \mathrm{FBS}$, rhM-CSF $(10 \mathrm{ng} / \mathrm{ml})$, and rhRANKL $(50 \mathrm{ng} / \mathrm{ml})$. To evaluate the effect of bortezomib (A), and immunomodulatory compounds (CC-4047, CC-6032, and CC-5013) (B) on osteoclast differentiation, these components were added to the culture. Media containing bortezomib (1.0$10 \mathrm{nM}$ ) were replenished twice a week, and immunomodulatory compounds $(10 \mu \mathrm{M})$ were added every day. TRAP staining was performed at day 14 , TRAP $^{+}$multinucleated cells $(>2)$ were counted as mature osteoclasts. The effects of bortezomib were different in PBMCs samples from two healthy donors (A). As the effects of immunomodulatory compounds showed similar results a representative result is shown (B). Results are expressed as the means \pm SEM of double experiments. dependent manner in osteoprogenitor cell culture medium, independent of treatment with bortezomib.

Both bortezomib (PS-341) and immunomodulatory thalidomide analogs suppress osteoclast differentiation. Next we investigated the effects of bortezomib and immunomodulatory compounds on osteoclast differentiation by TRAP staining. First, we cultured monocytes from $\mathrm{PB}$ in medium containing recombinant human M-CSF for 2 days. Subsequently, we cultured the cells with rhM-CSF, rhRANKL, and bortezomib

or immunomodulatory compounds. TRAP staining was performed at day 14. As shown in Fig. 6A, in culture using PBMCs from one healthy donor, 10 and $5.0 \mathrm{nM}$ bortezomib suppressed osteoclast differentiation, but there was no significant difference in TRAP-positive cells with 2.5 and $1.0 \mathrm{nM}$ bortezomib compared with the control. However, in culture using another healthy donor, even $1.0 \mathrm{nM}$ bortezomib suppressed osteoclast differentiation. On the other hand, the effects of immunomodulatory compounds on osteoclast differentiation showed similar results in two healthy donors; each compound suppressed osteoclast differentiation in $10 \mu \mathrm{M}$ (Fig. 6B). Therefore, both bortezomib and immunomodulatory compounds showed suppressive effects on osteoclast differentiation at anti-myeloma cell effect concentrations of 5.0 and $10 \mu \mathrm{M}$, respectively.

\section{Discussion}

Recent new drugs such as proteasome inhibitor, bortezomib (PS-341) and lenalidomide are improving the complete response rate and OS for patients with MM (17-24), and recent studies revealed the efficacy of immunomodulatory compounds for drug-resistant myeloma cells $(15,25-27)$. First, regarding the proteasome inhibitor, bortezomib, some reports have shown an increase of ALP activity after bortezomib administration $(8,9,28)$, and one study in mice revealed that treatment with proteasome inhibitors promoted bone formation and increased BMP2 expression (10). On the other hand, an up-to-date study revealed inhibition of osteolastogenesis by bortezomib (29). As for immunomodulatory thalidomide analogs, CC-4047 was reported to inhibit osteoclast formation by down-regulation of PU.1 (30), and Revlimid (CC-5013, lenalidomide) was thought to inhibit osteoclast growth and survival (Breitkreutz et al, ASCO, abs. 7606, 2006). Although immunomodulatory compounds are known to inhibit interaction between myeloma cells and BM stromal cells (osteoblasts) (31), the direct effect of immunomodulatory compounds on osteoblasts is not clear.

For the investigation of osteoblast differentiation, we used an hMSC culture system. Although recent studies pointed out the abnormal features of MSCs in MM patients $(32,33)$, we thought that the BM milieu with the proliferation of myeloma cells produced abnormal characteristics of MSCs. We added 0.5-5 nM bortezomib to an osteoprogenitor cell culture based on data showing the range of $\mathrm{IC}_{50}$ to be $2.5-30 \mathrm{nM}$ when bortezomib was administered to patients with MM (14) and our preliminary result showed that $10 \mathrm{nM}$ bortezomib was too cytotoxic for osteoprogenitor cells (data not shown). Subsequently, we changed the concentration or the time of bortezomib addition to the culture media to investigate how 
they would work. In contrast, we decided to add $10 \mu \mathrm{M}$ of each immunomodulatory compound to the medium for osteoprogenitor cell culture because this concentration showed a cytotoxic effect on myeloma cells (Fig. 1B), as in the study by Hideshima et al (15). Here we determined ALP activity as an indicator of a differentiation marker of osteoblasts from the early to mature phase, and mineralized nodule formation detected by von Kossa staining as an indicator of ultimately differentiated osteoblasts.

First, $5.0 \mathrm{nM}$ botrtezomib showing clear cytotoxicity to myeloma cell lines suppressed ALP activity, and $2.5 \mathrm{nM}$ bortezomib also suppressed ALP activity when it was added to the media from the initiation of osteoprogenitor cell culture medium; however, this concentration did not suppress ALP activity compared with the control when it was added 5 days after the initiation of this medium. Furthermore, ALP activity increased according to the decrease of bortezomib concentration $(1.0$ and $0.5 \mathrm{nM})$. It was doubtful whether these low concentrations of bortezomib would show an antimyeloma cell effect, and in fact these concentrations were not effective on myeloma cell lines or patient myeloma cells (Fig. 1). The result of the anti-myeloma cell effect of bortezomib requiring a high concentration seemed to be contradictory to the promotion of osteoblast differentiation by low concentration in clinical administration; however, $2.5 \mathrm{nM}$ bortezomib induced apoptosis in primary myeloma cells and, considering that our cell proliferation assay was performed in $10 \%$ FCS, we expected that an effective bortezomib concentration in sera of MM patients could not be fixed. In contrast, von Kossa staining at day 42 after the initiation of osteoprogenitor cell culture showed mineralized nodule formation with $2.5 \mathrm{nM}$ bortezomib; therefore, we could not state that $2.5 \mathrm{nM}$ bortezomib suppressed osteoblast differentiation in the long term.

As for the expression of BMP2, the addition of bortezomib to media did not increase its expression, and BMP2 expression might depend on the duration of culture from the initiation of osteoprogenitor cell culture to RNA extraction. Considering that the recent study by Giuliani et al (34) showed that bortezomib significantly increased transcription factor Runx2/Cbfa1 activity by the ELISA-based method and immunostaining performed on BM biopsies, the expression of BMP2 protein should have been analyzed later by similar methods.

Next, the effective concentration of bortezomib to suppress osteoclast differentiation might differ among cases. In one healthy donor sample, $<2.5 \mathrm{nM}$ bortezomib did not suppress osteoclast differentiation; however, even $1.0 \mathrm{nM}$ bortezomib suppressed it in another case, as well as in the result reported by von Metzler et al (29). Surprisingly, even $0.1 \mathrm{nM}$ bortezomib suppressed osteoclast differentiation in their study, and we would suggest that a high concentration $(5.0 \mathrm{nM})$ of bortezomib would certainly be effective in suppressing osteoclast differentiation. This concentration might be reasonable for the treatment of MM since it could be useful for both an antimyeloma cell effect and anti-osteoclast differentiation. Considering the relationship between myeloma cells and osteoclasts, in which osteoclasts support the survival and proliferation of myeloma cells (35), treatment with bortezomib in $\mathrm{MM}$ patients could be useful and reasonable. Therefore, if there was no direct anti-myeloma effect with a low concentration of bortezomib, we could expect an indirect anti-myeloma effect through the suppression of osteoclast differentiation.

Osteoblast differentiation was not suppressed even in $10 \mu \mathrm{M}$ immunomodulatory compounds, which showed strong cytotoxicity to myeloma cell lines (15), because ALP activity did not decrease and mineralized nodule formation was detected by von Kossa staining. On the other hand, we could expect a strong cytotoxic effect of immunomodulatory compounds on osteoclast differentiation.

In conclusion, it is expected that bortezomib in a range of concentrations would show both an anti-myeloma cell effect and promotion of bone formation in MM patients, and an anti-osteoclast effect at low concentrations in some cases. On the other hand, we could expect that immunomodulatory compounds would show both anti-myeloma cell and osteoclast effects at a concentration not cytotoxic to osteoblast differentiation. Therefore, we might improve the treatment strategy for MM patients without damage to BM stromal cells by combining bortezomib with immunomodulatory compounds such as lenalidomide.

\section{Acknowledgments}

We appreciate the help of H. Sumida, K. Yamamoto, N. Nakaju, S. Matsumoto, and R. Yamaguchi for their excellent technical or secretarial assistance.

\section{References}

1. Durie BGM and Salmon SE: A clinical staging system for multiple myeloma. Cancer 36: 842-854, 1975.

2. Ishikawa $\mathrm{H}$, Tanaka $\mathrm{H}$, Iwato $\mathrm{K}$, Tanabe $\mathrm{O}$, Asaoku $\mathrm{H}$, Nobuyoshi M, Yamamoto I, Kawano M and Kuramoto A: Effect of glucocorticoids on the biologic activity of myeloma cells: Inhibition of interleukin-1ß ostoclasts activating factor-induced bone resorption. Blood 75: 715-720, 1990.

3. Abe M, Hiura K, Wilde J, Moriyama k, Hashimoto T, Ozaki S, Wakatsuki S, Kosaka M, Kido S, Inoue D and Matsumoto T: Role for macrophage inflammatory protein (MIP)-1alpha and MIP-1beta in the development of osteolytic lesions in multiple myeloma. Blood 100: 2195-2202, 2002.

4. Pearse RN, Sordillo EM, Yaccoby S, Wong BR, Liau DF, Colman N, Michaeli J, Epstein J and Choi Y: Multiple myeloma disrupts the TRANCE/osteoprotegerin cytokine axis to trigger bone destruction and promote tumor progression. Proc Natl Acad Sci USA 98: 11581-11586, 2001.

5. Sezer O, Heider U, Zavrski I, Kuhne CA and Hofbauer LC: RANK ligand and osteoprotegerin in myeloma bone disease. Blood 101: 2094-2098, 2003.

6. Tian E, Zhan F, Walker R, Rasmussen E, Ma Y, Barlogie B and Shaughnessy JD: The role of Wnt-signaling antagonist DKK1 in the development of osteolytic lesions in multiple myeloma. $\mathrm{N}$ Engl J Med 349: 2483-2494, 2003.

7. Oshima T, Abe M, Asano J, Hara T, Kitazoe K, Sekimoto E, Tanaka Y, Shibata H, Hashimoto T, Ozaki S, Kido S, Inoue D and Matsumoto T: Myeloma cells suppress bone formation by secreting a soluble Wnt idnhibitor, sFRP-2. Blood 106: 3160-3165, 2005.

8. Shimazaki C, Uchida R, Nakano S, Namura K, Fuchida SI, Okano A, Okamoto $\mathrm{M}$ and Inaba T: High serum bone-specific alkaline phosphatase level after bortezomib-combined therapy in refractory multiple myeloma: possible role of bortezomib on osteoblast differentiation. Leukemia 19: 1102-1103, 2005.

9. Zangari M, Esseltine D, Lee CK, Barlogie B, Elice F, Burns MJ, Kang SH, Yaccoby S, Najarian K, Richardson P, Sonneveld P and Tricot G: Response to bortezomib is associated to osteoblastic activation in patients with multiple myeloma. $\mathrm{Br} \mathrm{J}$ Haematol 131: 71-73, 2005 . 
10. Garrett IR, Chen D, Gutierrez G, Zhao M, Escobedo A, Rossini G, Harris SE, Gallwitz W, Kim KB, Hu S, Crews CM and Mundy GR: Selective inhibitors of the osteoblast proteasome stimulate bone formation in vivo and in vitro. J Clin Invest 111: 1771-1782, 2003

11. Sakai A, Marti GE, Caporaso N, Pittaluga S, Touchman JW, Fend $\mathrm{F}$ and Raffeld $\mathrm{M}$ : Analysis of expressed immunoglobulin heavy chain genes in familial B-CLL. Blood 95: 1413-1419, 2000.

12. Ishiyama M, Tominaga H, Shiga M, Sasamoto K, Ohkura Y and Ueno $\mathrm{K}$ : A combined assay of cell viability and in vitro cytotoxicity with a highly water-soluble tetrazolium salt, neutral red and crystal violet. Biol Pharm Bull 19: 1518-1520, 1996.

13. Takayanagi H, Ogasawara K, Hida S, Chiba T, Murata S, Sato K, Takaoka A, Yokochi T, Oda H, Tanaka K, Nakamura K and Taniguchi T: T-cell-mediated regulation of osteoclastogenesis by signaling cross-talk between RANKL and IFN-gamma. Nature 30: 600-605, 2000.

14. Hideshima T, Richardson P, Chauhan D, Palombella VJ, Elliott PJ, Adams J and Anderson KC: The proteasome inhibitor PS-341 inhibits growth, induces apoptosis, and overcomes drug resistance in human multiple myeloma cells. Cancer Res 61: 3071-3076, 2001.

15. Hideshima T, Chauhan D, Shima Y, Raje N, Davies FE, Tai YT, Treon SP, Lin B, Schlossman RL, Richardson P, Muller G, Stirling DI and Anderson KC: Thalidomide and its analogs overcome drug resistance of human multiple myeloma cells to conventional therapy. Blood 96: 2943-2950, 2000.

16. Luo Q, Kang Q, Si W, Jiang W, Park JK, Peng Y, Li X, Luu HH, Luo J, Montag AG, Haydon RC and He TC: Connective tissue growth factor (CTGF) is regulated by Wnt and bone morphogenetic proteins signaling in osteoblast differentiation of mesenchymal stem cells. J Biol Chem 279: 55958-55968, 2004.

17. Richardson PG, Sonneveld P, Schuster MW, Irwin D, Stadtmauer EA, Facon T, Harousseau JL, Ben-Yehuda D, Lonial S, Goldschmidt H, Reece D, San-Miguel JF, Bladé J, Boccadoro M, Cavenagh J, Dalton WS, Boral AL, Esseltine DL, Porter JB, Schenkein D and Anderson KC: Assessment of proteasome inhibition for extending remissions (APEX) investigators. Bortezomib or high-dose dexamethasone for relapsed multiple myeloma. N Engl J Med 352: 2487-2498, 2005.

18. Mateos MV, Hernández JM, Hernández MT, Gutiérrez NC, Palomera L, Fuertes M, Díaz-Mediavilla J, Lahuerta JJ, De la Rubia J, Terol MJ, Sureda A, Bargay J, Ribas P, De Arriba F, Alegre A, Oriol A, Carrera D, García-Laraña J, García-Sanz R, Bladé J, Prósper, F, Mateo G, Esseltine DL, van de Velde H and San Miguel JF: Bortezomib plus melphalan and prednisone in elderly untreated patients with multiple myeloma: results of a multicenter phase 1/2 study. Blood 108: 2165-2172, 2006.

19. Barlogie B, Tricot G, Anaissie E, Shaughnessy J, Rasmussen E, van Rhee F, Fassas A, Zangari M, Hollmig K, Pineda-Roman M, Lee C, Talamo G, Thertulien R, Kiwan E, Krishna S, Fox M and Crowley J: Thalidomide and hematopoietic-cell transplantation for multiple myeloma. N Engl J Med 354: 1021-1030, 2005.

20. Cavo M: Proteasome inhibitor bortezomib for the treatment of multiple myeloma. Leukemia 20: 1341-1352, 2006.

21. Palumbo A, Bringhen S, Caravita T, Merla E, Capparella V, Callea V, Cangialosi C, Grasso M, Rossini F, Galli M, Catalano L, Zamagni E, Petrucci MT, De Stefano V, Ceccarelli M, Ambrosini MT, Avonto I, Falco P, Ciccone G, Liberati AM, Musto P and Boccadoro M: Italian Multiple Myeloma Network, GIMEMA. Oral melphalan and prednisone chemotherapy plus thalidomide compared with melphalan and prednisone alone in elderly patients with multiple myeloma: randomised controlled trial. Lancet 367: 825-831, 2006.
22. Nencioni A, Grünebach F, Patrone F, Ballestrero A and Brossart P: Proteasome inhibitors: antitumor effects and beyond. Leukemia 21: 30-36, 2007.

23. Barlogie B, Anaissie E, van Rhee F, Haessler J, Hollmig K, Pineda-Roman M, Cottler-Fox M, Mohiuddin A, Alsayed Y, Tricot G, Bolejack V, Zangari M, Epstein J, Petty N, Steward D, Jenkins B, Gurley J, Sullivan E, Crowley J and Shaughnessy JD: Incorporating bortezomib into upfront treatment for multiple myeloma: early results of total therapy 3 . Br J Haematol 138: 176-185, 2007.

24. Jagannath S, Richardson PG, Sonneveld P, Schuster MW, Irwin D, Stadtmauer EA, Facon T, Harousseau JL, Cowan JM and Anderson KC: Bortezomib appears to overcome the poor prognosis conferred by chromosome 13 deletion in phase 2 and 3 trials. Leukemia 21: 151-157, 2007.

25. Mitsiades N, Mitsiades CS, Poulaki V, Chauhan D, Richardson PG, Hideshima T, Munshi NC, Treon SP and Anderson KC: Apoptotic signaling induced by immunomodulatory thalidomide analogs in human multiple myeloma cells: therapeutic implications. Blood 99: 4525-4530, 2002.

26. Richardson PG, Schlossman RL, Weller E, Hideshima T, Mitsiades C, Davies F, LeBlanc R, Catley LP, Doss D, Kelly K, McKenney M, Mechlowicz J, Freeman A, Deocampo R, Rich R, Ryoo JJ, Chauhan D, Balinski K, Zeldis J and Anderson KC: Immunomodulatory drug CC-5013 overcomes drug resistance and is well tolerated in patients with relapsed multiple myeloma. Blood 100: 3063-3067, 2002.

27. Teo SK: Properties of thalidomide and its analogues: implications for anticancer therapy. The AAPS J 7: E14-E19, 2005.

28. Heider U, Kaiser M, Muller C, Jakob C, Zavrski I, Schulz CO, Fleissner C, Hecht M and Sezer O: Bortezomib increases osteoblasts activity in myeloma patients irrespective of response to treatment. Eur J Haematol 77: 233-238, 2006.

29. Von Metzler I, Krebbel H, Hecht M, Manz RA, Fleissner C, Mieth M, Kaiser M, Jakob C, Sterz J, Kleeberg L, Heider U and Sezer O: Bortezomib inhibits human osteoclastogenesis. Leukemia 21: 2025-2034, 2007.

30. Anderson G, Gries M, Kurihara N, Honjo T, Anderson J, Donnenberg V, Donnenberg A, Ghobrial I, Mapara MY, Stirling D, Roodman D and Lentzsch S: Thalidomide derivative CC-4047 inhibits osteoclast formation by down-regulation of PU.1. Blood 107: 3098-3105, 2006.

31. Hideshima T and Anderson KC: Molecular mechanisms of novel therapeutic approaches for multiple myeloma. Nat Rev 2: 927-937, 2002.

32. Corre J, Mahtouk K, Attal M, Gadelorge M, Huynh A, FleuryCappellesso S, Danho C, Laharrague P, Klein B, Rème T and Bourin P: Bone marrow mesenchymal stem cells are abnormal in multiple myeloma. Leukemia 21: 1079-1088, 2007.

33. Arnulf B, Lecourt S, Soulier J, Ternaux B, Lacassagne MN, Crinquette A, Dessoly J, Sciaini AK, Benbunan M, Chomienne C, Fermand JP, Marolleau JP and Larghero J: Phenotypic and functional characterization of bone marrow mesenchymal stem cells derived from patients with multiple myeloma. Leukemia 21: 158-163, 2007.

34. Giuliani N, Morandi F, Tagliaferri S, Lazzaretti M, Bonomini S, Crugnola M, Mancini C, Martella E, Ferrari L, Tabilio A and Rizzoli V: The proteasome inhibitor bortezomib affects osteoblast differentiation in vitro and in vivo in multiple myeloma patients. Blood 110: 334-338, 2007.

35. Abe M, Hiura K, Wilde J, Moriyama K, Hashimoto T, Ozaki S, Wakatsuki S, Kosaka M, Kido S, Inoue D and Matsumoto T: Osteoclasts enhance myeloma cell growth and survival via cell-cell contact: a vicious cycle between bone destruction and myeloma expansion. Blood 104: 2484-2491, 2004. 